\title{
Preclinical progress and first translational steps for a liposomal chemotherapy protocol against adrenocortical carcinoma
}

\author{
Sara Jung', Zoltan Nagy², Martin Fassnacht3,4, Gerard Zambetti5, Max Weiss6, \\ Martin Reincke', Peter Igaz², Felix Beuschlein' ${ }^{1}$ and Constanze Hantel' \\ ${ }^{1}$ Endocrine Research Unit, Medizinische Klinik und Poliklinik IV, Ludwig-Maximilians-University, Munich, Germany \\ 22nd Department of Medicine, Semmelweis University, Faculty of Medicine, Budapest, Hungary \\ 3Department of Internal Medicine I, Division of Endocrinology and Diabetes, University Hospital, \\ University of Würzburg, Würzburg, Germany \\ ${ }^{4}$ Comprehensive Cancer Center Mainfranken, University of Würzburg, Würzburg, Germany \\ ${ }^{5}$ Department of Pathology, St Jude Children's Research Hospital, Memphis, Tennessee, USA \\ 6Institute of Pathology, Ludwig-Maximilians-University, Munich, Germany
}

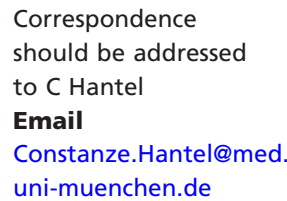

\begin{abstract}
Systemic therapy of adrenocortical carcinoma (ACC) is limited by heterogeneous tumor response and adverse effects. Recently, we demonstrated anti-tumor activity of LEDP-M (etoposide, liposomal doxorubicin, liposomal cisplatin, mitotane), a liposomal variant of EDP-M (etoposide, doxorubicin, cisplatin, mitotane). To improve the therapeutic efficacy and off-target profiles of the clinical gold standard EDP-M, we investigated liposomal EDP-M regimens in different preclinical settings and in a small number of ACC patients with very advanced disease. Short- and long-term experiments were performed on two ACC models (SW-13 and SJ-ACC3) in vivo. We evaluated the anti-tumoral effects and off-target profiles of EDP-M, LEDP-M and a novel regimen L(I)EDP-M including liposomal etoposide. Furthermore, the role of plasma microRNA-210 as a therapeutic biomarker and first clinical data were assessed. Classical and liposomal protocols revealed anti-proliferative efficacy against SW-13 (EDP-M $P<0.01$; LEDP-M: $P<0.001$; L(I)EDP-M: $P<0.001$ vs controls), whereas in SJ-ACC3, only EDP-M ( $P<0.05$ vs controls) was slightly effective. Long-term experiments in SW-13 demonstrated anti-tumor efficacy for all treatment schemes (EDP-M: $P<0.01$, LEDP-M: $P<0.05$, L(I)EDP-M $P<0.001$ vs controls). The analysis of pre-defined criteria leading to study termination revealed significant differences for control $(P<0.0001)$ and EDP-M $(P=0.003)$ compared to L(I)EDP-M treatment. Raising its potential for therapy monitoring, we detected elevated levels of circulating microRNA-210 in SW-13 after LEDP-M treatment $(P<0.05)$. In contrast, no comparable effects were detectable for SJ-ACC3. However, overall histological evaluation demonstrated improved off-target profiles following liposomal regimens. The first clinical data indicate improved tolerability of liposomal EDP-M, thus confirming our results. In summary, liposomal EDP-M regimens represent promising treatment options to improve clinical treatment of ACC.
\end{abstract}

\author{
Key Words \\ - adrenocortical \\ carcinoma \\ - liposomal doxorubicin \\ - liposomal cisplatin \\ - liposomal etoposide \\ - Caelyx \\ - Lipoplatin \\ - Myocet \\ - NCl-H295R \\ - SJ-ACC3 \\ - SW-13 \\ - microRNA-210 \\ - microRNA-483-5p
} (2016) 23, 825-837 


\section{Introduction}

In recent years, genetic and molecular profiling of surgical tumor specimens has led to the identification of novel biomarkers with prognostic relevance for patients with adrenocortical carcinoma (ACC) (Assie et al. 2014). However, the complex interplay of underlying pathways that relate to treatment response remains largely unknown (Zheng et al. 2016). Accordingly, targeted therapies in the treatment of ACC patients have shown limited efficacy, and clinical management is mainly restricted to common cytotoxic agents (Berruti et al. 2012, Kroiss et al. 2012, Fassnacht et al. 2015). The current mainstay of treatment for advanced and metastasized ACC is defined as the EDP-M protocol (Fassnacht et al. 2012) consisting of classical formulations of the chemotherapeutic agents etoposide (E), doxorubicin (D), cisplatin (P) and mitotane (M). However, the overall therapeutic efficacy of this treatment scheme is still dissatisfactory, and the combination of these chemotherapeutic agents is associated with severe and dose-limiting adverse effects (Fassnacht et al. 2012). Moreover, therapeutic biomarkers are yet to be defined to identify the patients with relevant therapeutic benefit of this highly toxic regimen (Ronchi et al. 2009, Malandrino et al. 2010).

In the past years, encapsulation of doxorubicin and cisplatin in liposomes has been demonstrated as an important strategy for enhancing therapeutic efficacy and improving the off-target profiles of the parental drugs mainly regarding cardiotoxicity and nephrotoxicity (Boulikas 2009, Gabizon et al. 2012). Recently, our group has demonstrated furthermore an extraordinary uptake phenomenon of liposomes specifically by adrenocortical tumor cells (Hantel et al. 2010, 2012). Moreover, we provided evidence in a commonly used xenograft model for ACC (NCI-H295R) that an exchange of doxorubicin and cisplatin by liposomal variants (LEDP-M) was associated with enhanced therapeutic efficacy and reduced adverse effects (Hantel et al. 2014). Of note, liposomal formulations of doxorubicin (Myocet and Caelyx) or cisplatin (Lipoplatin) are already in clinical use for a wide range of malignancies including metastatic breast cancer, advanced ovarian cancer, progressive multiple myeloma, AIDS-related Kaposi's sarcoma or pancreatic cancer and non-small-cell lung cancer, respectively (Boulikas 2009, Marchal et al. 2015).

However, clinical translation of novel regimens for ACC emerged as challenging, particularly due to observed heterogeneity of ACC patients regarding tumor growth rate, treatment response and overall survival. Thus, we investigated LEDP-M in two further xenograft models for ACC in this study: SW-13 and the recently introduced pediatric xenograft model SJ-ACC3 (Leibovitz et al. 1973, Pinto et al. 2013, Hantel \& Beuschlein 2016). Moreover, we included liposomal etoposide resulting in a novel treatment scheme now named L(l)EDPM. In addition, based on the detected differences in therapeutic responsiveness for both tumor models, we studied intratumoral and plasma microRNAs (miRs) as potentially interesting therapeutic biomarkers for ACC. Finally, we provide preliminary clinical data indicating that management of ACC patients, involving liposomal chemotherapies, might be a favorable clinical option to improve tolerability of the current gold-standard treatment for ACC.

\section{Materials and methods}

\section{Cell culture}

Material for cell culture was purchased from Gibco Invitrogen if not stated otherwise. SW-13 cells (obtained from ATCC and recently authenticated) were cultured in DMEM/F-12 medium (Dulbecco's Modified Eagle Medium) in a $5 \% \mathrm{CO}_{2}-95 \%$ air atmosphere at $37^{\circ} \mathrm{C}$. Cell culture medium was supplemented with $1 \%$ penicillin/streptomycin and 10\% FBS (heat-inactivated fetal bovine serum).

\section{Animal experiments}

Animals and preclinical ACC tumor models All animal experiments were approved by the Regierung von Oberbayern and are in accordance with the German guidelines for animal studies. Athymic NMRI $n u / n u$ mice (female, 6-8 weeks old) were purchased from Harlan Winkelmann (Borchen, Germany) and stored under pathogen-free conditions. For SW13-xenografts, tumor cells were inoculated in $200 \mu \mathrm{L}$ PBS (Gibco Invitrogen) for tumor induction. For therapeutic experiments, SW-13 cells were subcutaneously injected into the neck of each mouse (short term: $13 \times 10^{6}$ cells and long term: $11 \times 10^{6}$ cells/mouse). Cryopreserved SJ-ACC3 samples (Pinto et al. 2013) were subcutaneously engrafted as $2 \mathrm{~mm}^{3}$ tumor specimens; before implantation, they were transferred from liquid nitrogen to a $37^{\circ} \mathrm{C}$ water bath after which tumor tissue was rinsed several times in medium 199 (Gibco Invitrogen) supplemented with 1\% penicillin/ streptomycin (Gibco Invitrogen). Therapeutic treatments started for SW-13 in short-term experiments ( $n=7-8$ mice) at day 14 and in the long-term setting ( $n=14$ mice) at day 4 after tumor induction. For SJ-ACC3, short-term

Published by Bioscientifica Ltd. 
( $n=4-6$ mice) and long-term ( $n=5$ mice) therapeutic treatments were started several weeks after implantation to enable successful tumor engraftment.

Therapeutic experiments For therapeutic experiments, a preclinically adapted EDP-M protocol (Fassnacht et al. 2012) was administered as already implemented in a recent study (Hantel et al. 2014). Mitotane powder (HRA-Pharma) was dissolved in sterile autoclaved corn oil and intraperitoneally injected at a daily dose of $300 \mathrm{mg} / \mathrm{kg}$ body weight over three consecutive days before cytostatic treatment. All treatments were carried out at 24-h intervals. Cytostatic drugs were intravenously injected in a $2 \mathrm{mg} / \mathrm{kg}$ dose of cisplatin and Lipoplatin (Regulon Inc, Athens, Greece) and $10 \mathrm{mg} / \mathrm{kg}$ doses of doxorubicin, liposomal doxorubicin (Caelyx; JanssenCilag GmbH, Neuss, Germany), etoposide and liposomal etoposide (Encapsula NanoSciences LLC, Nashville, TN, USA) according to the treatment modalities outlined for one therapeutic cycle (Fig. 1).

For short-term experiments, tumor-bearing mice were treated with one therapeutic cycle. Forty-eight hours after the last therapeutic intervention studies were terminated, the animals were killed. Then, the tumors were excised, immediately processed for paraffin embedding and in case of sufficient material, also, snap-frozen. Moreover, EDTA-blood was collected and centrifuged at $2000 \boldsymbol{g}$ at $4^{\circ} \mathrm{C}$ to obtain plasma samples. Frozen tumors and plasma samples were stored at $-80^{\circ} \mathrm{C}$.

For long-term therapeutic protocols, mice received repeated treatment cycles with a therapy-free interval of ten days. Animals were monitored daily, and tumor sizes were measured every second day (as tumor length $\times$ width $\left.\left(\mathrm{cm}^{2}\right)\right)$. Although for SJ-ACC3 the long-term study was terminated after the second cycle, SW-13 tumor-bearing mice received up to four therapeutic cycles to allow a more detailed investigation regarding the achievement of pre-defined endpoints (including tumor sizes and adverse effects) in the different treatment groups. In this setting, animals were killed when tumors reached a longest tumor diameter of $1.5 \mathrm{~cm}$ or when specified side effects (as body weight loss or pathologically changed phenotype as abnormal body posture) occurred. For additional immunohistochemical and histological analysis,
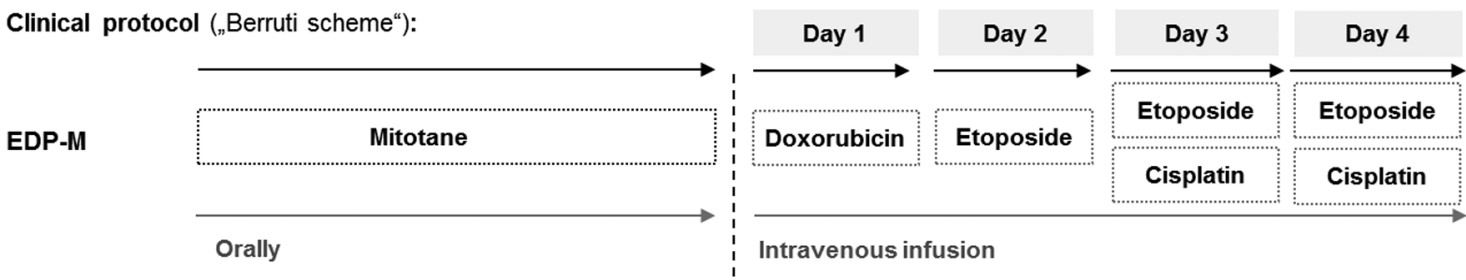

Preclinical protocol (one therapeutic cycle):
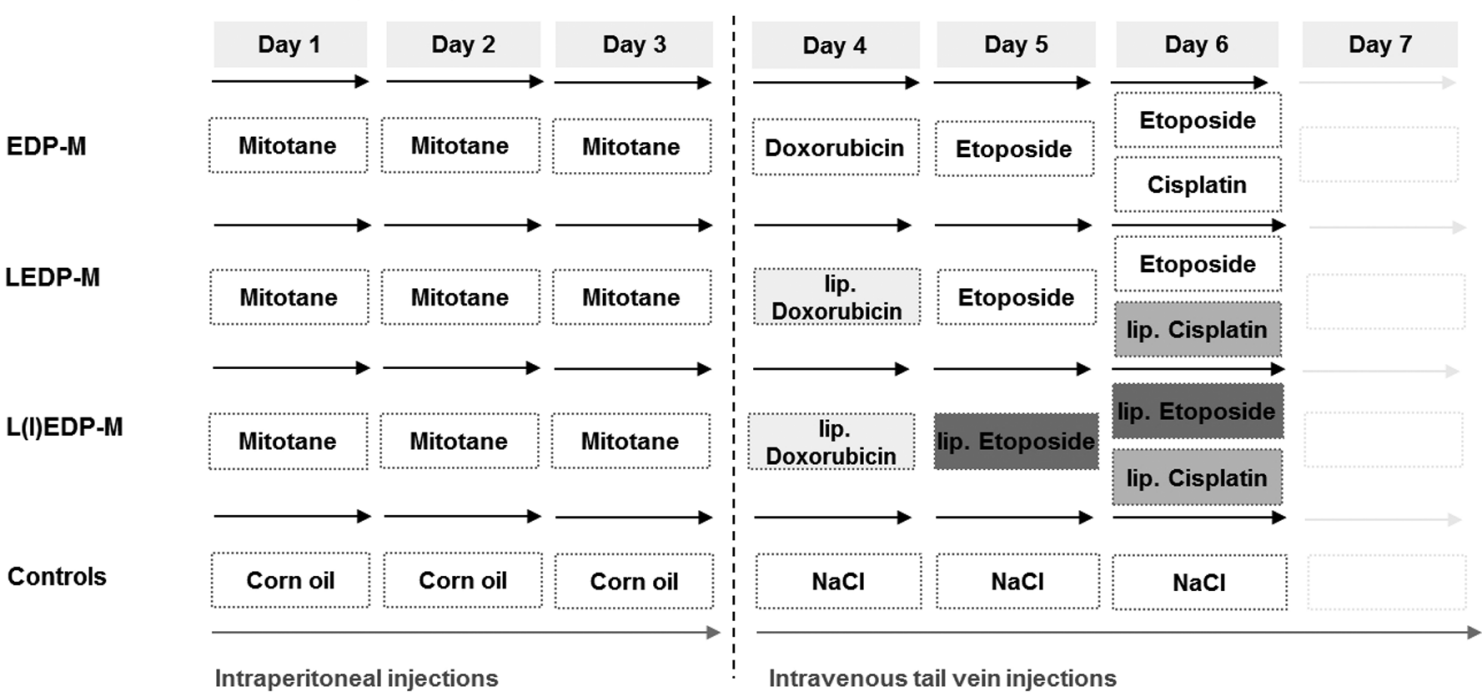

Figure 1

Schematic illustration of the preclinical treatment schemes EDP-M, LEDP-M and L(I)EDP-M adapted from the clinical EDP-M protocol exemplified for one therapeutic cycle. 
hearts and kidneys were also snap-frozen and paraffinembedded to enable a more detailed investigation of these main off-target organs.

\section{Histological and immunohistochemical} evaluation About $4 \mu \mathrm{m}$ sections (HM355E, Microm, Walldorf, Germany) of tumor tissue, kidneys and hearts were cut and rehydrated. Immunohistochemical staining of tumor tissue was performed after antigen retrieval using $10 \mathrm{mM}$ sodium citrate buffer, which was followed by incubation with blocking buffer containing 3\% BSA (Roche Diagnostics), $5 \%$ goat serum (Jackson ImmunoResearch) and $0.5 \%$ Tween 20 for $15 \mathrm{~min}$. Proliferating cells were stained using the monoclonal rabbit anti-human $\mathrm{Ki67}$ antibody (KI68R06, DCS innovative diagnostics, Hamburg, Germany) 1:200 in a blocking buffer. After overnight incubation at $4^{\circ} \mathrm{C}$, slides were rinsed three times for $5 \mathrm{~min}$ in PBS. Secondary polyclonal antibody (BA-1000 goat antirabbit biotinylated IgG, Vector Laboratories, Burlingame, CA, USA) was applied for $30 \mathrm{~min}$ at room temperature. Vectastain ABC Kit (Vector Laboratories) according to the manufacturer's protocol visualized bound primary antibody after $30 \mathrm{~min}$ followed by 3,3'-diaminobenzidine (Sigma-Aldrich) incubation. For counterstaining, Vector Methyl Green nuclear counterstain (Vector Laboratories) was applied for $10 \mathrm{~min}$ at $60^{\circ} \mathrm{C}$ to enable cell number quantification of Ki67-positive and-negative cells. Apoptotic cells were detected using the colorimetric DeadEnd TUNEL System (Promega) as described in the manufacturer's protocol. Tumors were immunohistochemically evaluated by investigating six high-power fields (HPF, $0.391 \mathrm{~mm}^{2}$, $400 \times$ magnification) per tumor. Histological evaluation of kidney and heart sections was performed using regressive hematoxylin/eosin (HE) staining (Harris Hematoxylin, Eosin Y, Sigma-Aldrich).

\section{Molecular analyses}

Based on a pilot experiment analyzing a panel of various miRs (miR-195, miR-210, miR-483-3p, miR-483-5p and miR-503) on NaCl, EDP-M and LEDP-M-treated NCIH295R xenografts (data not shown) and data from the literature, we selected two miRs (miR-483-5p and miR-210) for detailed examination in our recent study. MicroRNA (miR) was analyzed with real-time quantitative PCR (RT-qPCR) using short-term plasma and tumor samples of SW-13 and SJ-ACC3 mice. Circulating RNA from plasma exosomes was extracted by processing the plasma samples according to the manufacturer's protocols (Total Exosome Isolation Kit and Total Exosome RNA and Protein Isolation Kit, ThermoFisher Scientific) with addition of spike-in control cel-miR-39 (Ref. 2594091, Qiagen). Tumor RNA isolation, reverse transcription and RT-qPCR were performed as described previously (Nagy et al. 2015). Reactions were run in duplicates on a 96-well plate. Human primer assays and catalogue numbers (ThermoFisher Scientific) were as follows: Hsa-miR-210 (\#000512) and hsa-miR-483-5p (\#002338). For tumor and for plasma samples, RNU44 (\#001094) and cel-mir-39 (\#000200) were used as housekeeping genes, respectively (Brase et al. 2010, Nagy et al. 2015). miR levels were analyzed using the comparative $\Delta \mathrm{Ct}$ method (Schmittgen $\&$ Livak 2008). For evaluation of changes in miR level upon $\mathrm{NaCl}$ or LEDP-M treatment in individual mice, ratio of intratumoral to circulating miR level was calculated (miR ratio) after normalization to controls (\% of controls):

$$
\frac{\text { intratumoral } \mathrm{miR}(\%)}{\text { circulating } \mathrm{miR}(\%)}=\mathrm{miR} \text { ratio (individual animal) }
$$

A value of 1 indicates unchanged levels in tumor and plasma. A value $>1$ reveals an elevated expression of intratumoral miR, whereas a ratio $<1$ indicates elevated expression of circulating miR.

\section{Clinical data}

Six patients with very advanced ACC who have been benefited in the past from EDP-M, but experienced toxicity and/or desired experimental therapy, were offered liposomal doxorubicin or liposomal cisplatin (referred as liposomal EDP-M, lipEDP-M) on a compassionateuse basis (Table 1). This study was approved by the local ethic committee. All patients were informed about the experimental nature of this drug administration, and they gave informed consent in verbal and written forms.

\section{Statistical analysis}

Data are expressed as mean \pm s.E.m. (standard error of the mean). Analysis was performed with Prism (Houston, TX, USA) using the unpaired $t$-test or one-way analysis of variance (ANOVA) followed by Bonferroni's Multiple Comparison test (comparing all treatment groups) both including an adjustment of 95\% CI (confidence interval). For the analysis of pre-defined endpoints leading to study termination, log-rank (Mantel-Cox) test was applied using SPSS statistics 23 software. Statistical significance is defined as $P<0.05$ and is denoted as stars $\left({ }^{\star} P<0.05 ;{ }^{*} P<0.01\right.$; $\left.{ }^{* * *} P<0.001\right)$ in the figures if not stated otherwise.

Published by Bioscientifica Ltd. 


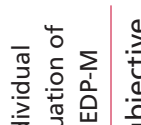

:

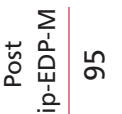

足

흔

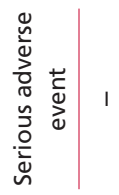

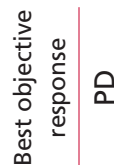

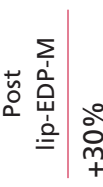

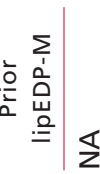

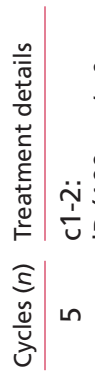

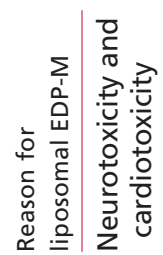

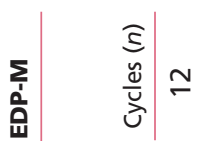

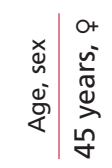

이

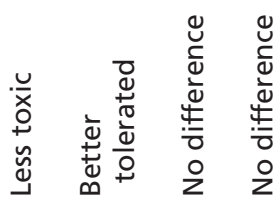

จ กิ กู่ คำ

$\infty$

$\hat{m} \stackrel{m}{\infty} \underset{\sigma}{\infty}$

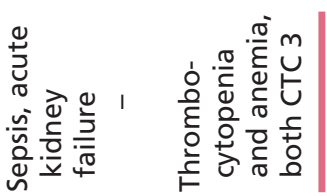

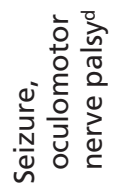

옹

옹

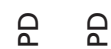

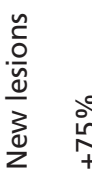

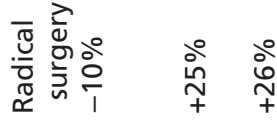

ஓ̊ำ

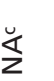

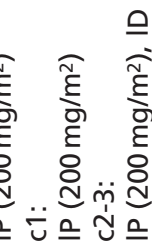

으 음

으

$\stackrel{\stackrel{\circ}{\sim}}{\stackrel{\circ}{+}} \stackrel{+}{+}$

$-\sim \sim N$

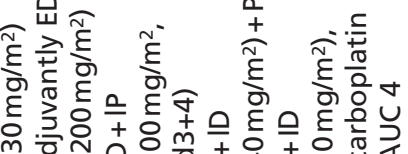

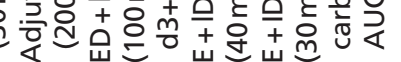

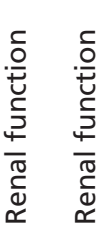

$\frac{\frac{1}{n}}{\frac{n}{3}}$

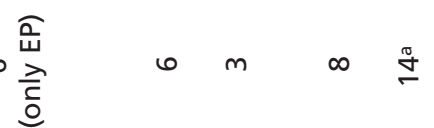

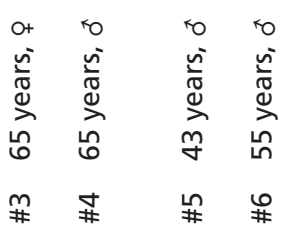

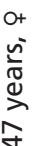

\#

$\frac{\frac{c}{5}}{\frac{n}{3}} \frac{\frac{\pi}{0}}{\frac{7}{2}}$

(n)

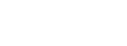




\section{Results}

\section{Investigation of short-term therapeutic efficacy on SW-13 and SJ-ACC3 xenografts}

In the first step, short-term therapeutic efficacies of three different treatment schemes EDP-M, LEDP-M and L(1) EDP-M were tested on SW-13 and SJ-ACC3 xenografts. After administration of one therapeutic cycle (Fig. 1), anti-tumoral effects were investigated regarding tumor cell proliferation and induction of apoptosis using Ki67 and TUNEL immunohistochemistry, respectively (Fig. 2).

For both xenograft models, the highest total cell number (Ki67-positive and -negative cells/high-power field/tumor) was documented in the tumors of the control group (SW-13: 28.9 \pm 2.2 ; SJ-ACC3: 35.9 \pm 1.3 ; Fig. 2A and C). Although all treatments showed antiproliferative effects against SW-13 (EDP-M: 20.5 \pm 1.6 , $P<0.01$; LEDP-M 17.2 $\pm 1.3, P<0.001$ and L(1)EDP-MP-M $14.7 \pm 0.9, P<0.001$, vs controls; Fig. 2A), for SJ-ACC3, only EDP-M led to a significant reduction in the number of tumor cells compared with controls (EDP-M: 30.3 \pm 1.2 , $P<0.05$; LEDP-M 31.5 $\pm 1.8, P>0.05$ and L(1)EDP-MP-M $32.5 \pm 0.3, P>0.05$ vs controls; Fig. 2C). Comparisons to other treatment groups were not significantly different $(P>0.05)$. For SW-13, the detected decrease in the number of tumor cells was furthermore accompanied by a severe condensation of nuclei upon specific treatments compared with controls (Fig. 2F, G, H and I). Moreover, these anti-tumoral effects were confirmed histologically by a semi-quantitative analysis of hematoxylin/eosinstained SW-13 tumors (Fig. 2J, K, L, M). Therefore, each tumor was histologically analyzed and categorized regarding necrotic areas (from low-to-high levels of necrosis with 0 to 3 , respectively). As shown in Fig. 2E, although necrosis was rarely detected for the control group, the presence and grades of necrosis increased upon EDP-M over LEDP-M to L(1)-EDP-M administration on SW-13 xenografts.

Quantification of apoptosis revealed no significant alterations $(P>0.05)$ upon specific therapeutic treatments (SW-13: controls 2.4 \pm 0.5 , EDP-M 3.0 \pm 0.5 , LEDP-M $4.2 \pm 0.7$ and L(l)EDP-MP-M 4.3 \pm 0.9 , Fig. 2B; SJ-ACC3: controls 3.5 \pm 0.8 , EDP-M 5.5 \pm 0.8 , LEDP-M 5.1 \pm 0.5 and L(1)EDP-MP-M 5.4 \pm 0.4 , Fig. 2D) for both tumor models.

\section{Investigation of long-term therapeutic efficacy on SW-13 and SJ-ACC3 xenografts}

Primary endpoint of the long-term study on SW-13 and SJ-ACC3 xenografts was the tumor size (expressed as length $\times$ width in $\mathrm{cm}^{2}$ ) upon repeated treatment with EDP-M, LEDP-M or L(1)EDP-M (Fig. 3). This experiment confirmed the previously obtained results
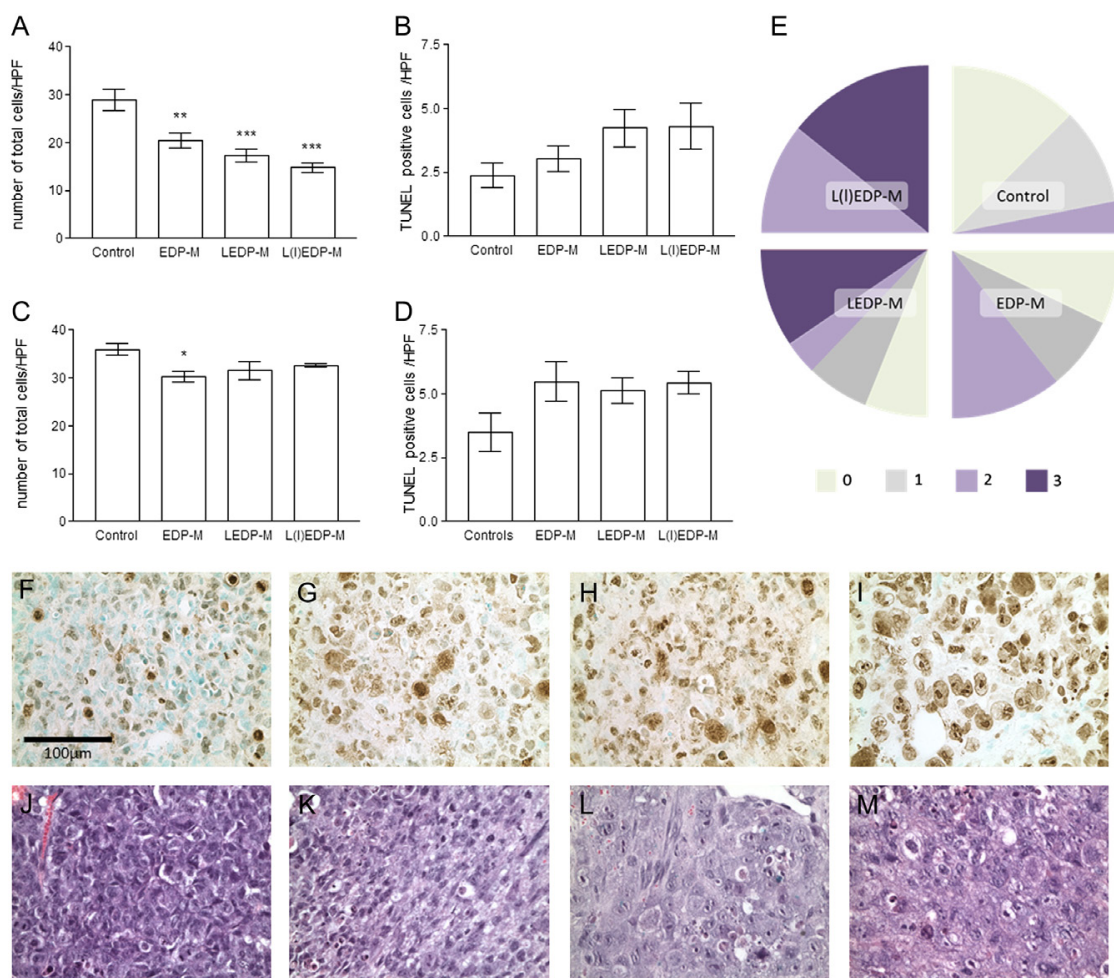

$E$

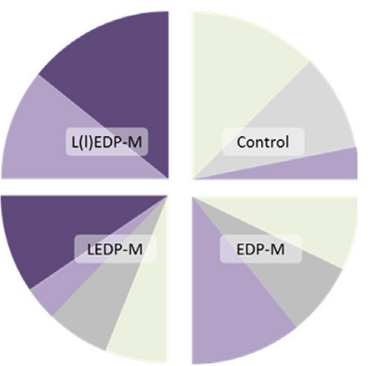

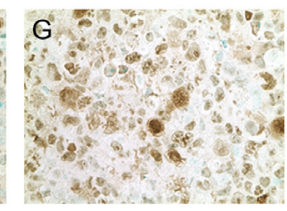
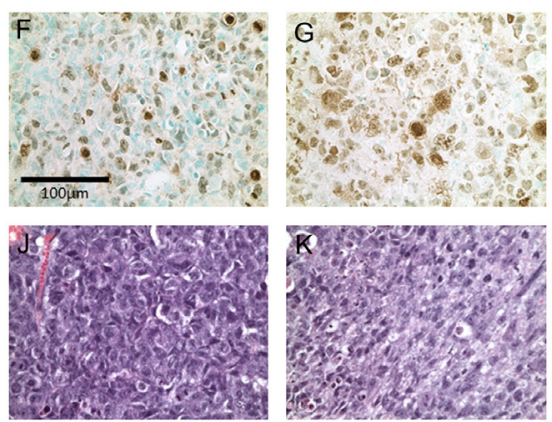

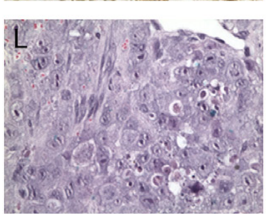

http://erc.endocrinology-journals.org DOI: 10.1530/ERC-16-0249
(C) 2016 Society for Endocrinology Printed in Great Britain
Published by Bioscientifica Ltd
Figure 2

Immunohistochemical and histological analysis of SW-13 (A, B and E) and SJ-ACC3 (C and D) xenografts derived from the short-term therapeutic experiment with quantification of Ki67-positive, Ki67-negative ( $A$ and $C$ ) and TUNEL-positive cells ( $B$ and D). Necrosis was analyzed semi-quantitatively using four categories ranked in ascending order from 0 (no necrosis) until 3 (extensive necrosis) for each SW-13 tumor, respectively (E). Representative pictures from Ki67 $(\mathrm{F}-\mathrm{I})$ and $\mathrm{H} \& \mathrm{E}$ staining $(\mathrm{J}-\mathrm{M})$ are presented for $\mathrm{NaCl}$ ( $\mathrm{F}$ and J), EDP-M (G and K), LEDP-M ( $\mathrm{H}$ and $\mathrm{L}$ ) and L(I)EDP-M (I and M)-treated SW-13 tumors. Statistical significance vs controls is denoted as stars $(* P<0.05 ; * * P<0.01 ; * * * P<0.001)$. 
from the short-term experiments for SW-13. The longterm study demonstrated anti-tumoral efficacy for all treatment modalities showing, in all regimens, a significant reduction in tumor size upon two therapeutic cycles compared with controls (day 29 after tumor cell injection, $\mathrm{NaCl}$ : $1.02 \pm 0.08$; EDP-M: $0.57 \pm 0.10$, $P<0.01 ; \quad$ LEDP-M $\quad 0.62 \pm 0.11, \quad P<0.05 ; \quad$ L(l)EDP-M: $0.48 \pm 0.07, P<0.001$, Fig. $3 A$ ). In contrast, for SJ-ACC3, no significant differences were detectable upon two cycles with the different therapeutic regimens (day 56 after implantation of tumor pieces, $\mathrm{NaCl}$ : $0.30 \pm 0.14$; EDP-M: $0.14 \pm 0.06, P>0.05$; LEDP-M 0.12 $\pm 0.03, P>0.05$; L(1)EDP-M: $0.17 \pm 0.08, P>0.05$; Fig. 3B).

\section{Investigation of tolerability and off-target profiles on long-term treatment}

In an attempt to investigate long-term tolerability of the different treatment modalities, we continued the therapeutic treatment of SW-13 tumor-bearing mice up to four cycles. Following this approach, we monitored the achievement of pre-defined endpoints such as $1.5 \mathrm{~cm}$ long tumor diameter or the appearance of severe adverse effects (body weight loss and ascites) for study termination. As shown in Fig. 3C, following these criteria, L(1)EDP-M treatment led to significantly prolonged time to reach these pre-defined criteria in comparison with not only controls $(P<0.0001)$ but also EDP-M $(P=0.003)$. Assessing the reason for study termination, severe adverse effects occurred in $43 \%$ of animals treated with classical EDP-M, whereas a liposomal treatment led to 0\% (LEDP-M) and only 7\% (L(1)EDP-M) cases of adverse effects (Fig. 3D).

We further investigated kidneys and hearts from treated animals as these organs represent main targets for side effects of classical formulations of cisplatin and doxorubicin. Although HE staining of kidneys from

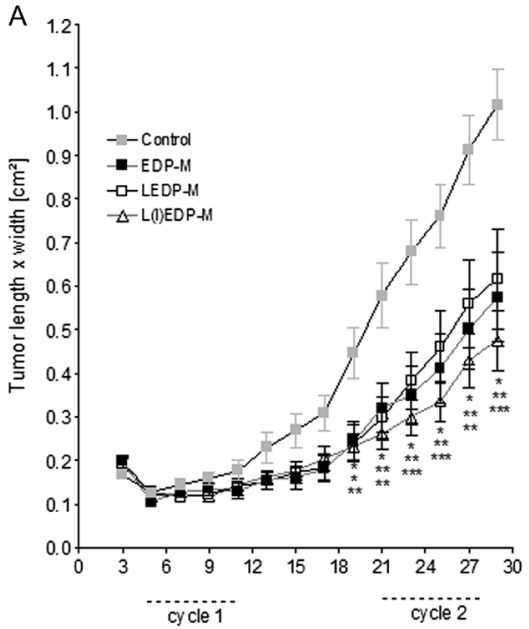

Time [Days after tumor induction]

C

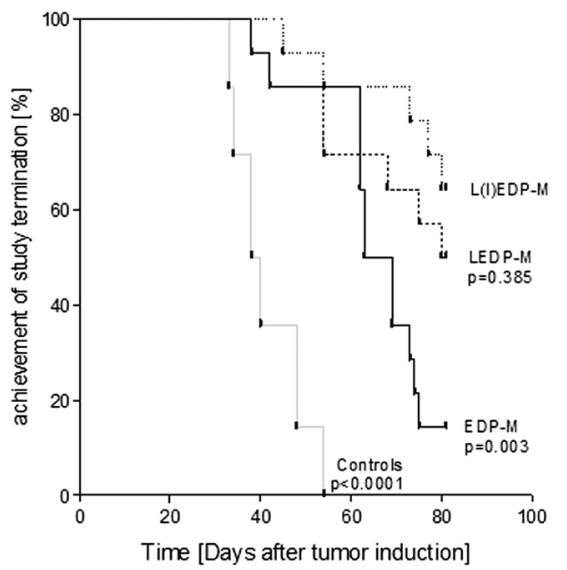

D

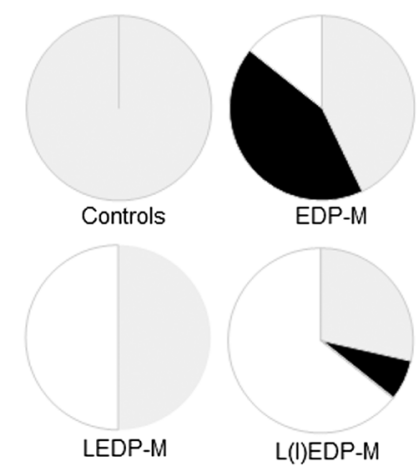

tumor diameter $\square$ study termination adverse-effects (body weight loss, in one case of the EDP-M group swollen abdomen)

Time [Days after tumor induction]

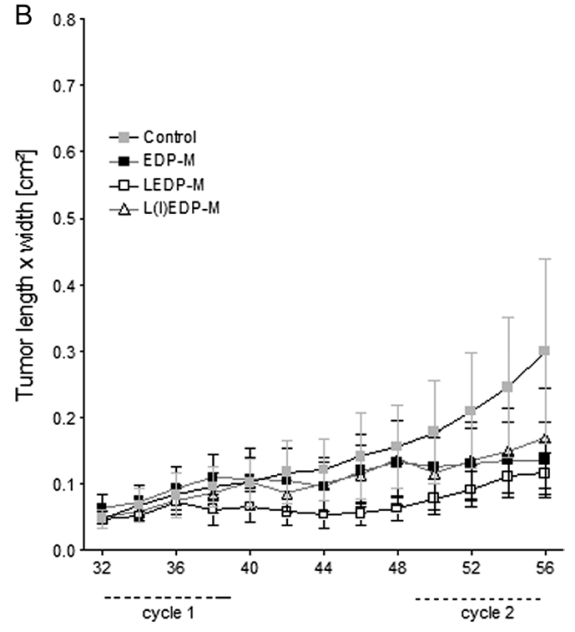

\section{Figure 3}

Effects on tumor size of SW-13 (A) and SJ-ACC3 (B) xenografts after two therapeutic cycles with the different treatment modalities. Statistical comparisons were performed using a one-way ANOVA followed by Bonferroni's Multiple Comparison Test (compare all groups). Stars $\left({ }^{*} P<0.05 ; * * P<0.01 ; * * * P<0.001\right)$ denote significant differences compared with controls, comparisons to other treatment groups were not significant $(P>0.05)$. SW-13 tumor-bearing mice that received up to four therapeutic cycles of $\mathrm{NaCl}$, EDP-M, LEDP-M or L(I)EDP-M treatment were killed after achieving pre-defined endpoints. Regarding the achievement of study termination, the comparison of all treatment groups resulted in significant differences using a log-rank (Mantel-Cox) analysis. Significant differences are illustrated in (C) in comparison to L(I)EDP-M. Individual reasons for study termination are furthermore depicted in (D). 
control mice $(n=14)$ did not reveal any pathological finding, 5 of 14 kidneys in the EDP-M group displayed severe pathologies in the form of tubular casts (Fig. 4). However, such histological changes were not detectable in the therapeutic arms using liposomal formulations of cisplatin. Specifically, in none of the 14 animals treated with LEDP-M and only 1 of 14 mice treated with L(1)EDP$\mathrm{M}$, minor structural renal changes were found. Moreover, mild cardiotoxic effects were also detectable in the EDP-M arm exclusively. Although we observed vacuole formation and single-cell necrosis in 2 of 5 investigated hearts of the EDP-M group, no such effects were apparent in the other treatment groups (Fig. 4).

\section{Preclinical investigation of circulating miR-210 as potential biomarker to monitor therapeutic efficacy}

After one therapeutic cycle with $\mathrm{NaCl}$ or LEDP-M, miR ratio revealed no treatment-dependent changes for miR-483-5p expression in both tumor models
(SW-13: control 1.38 \pm 0.52 , LEDP-M 0.81 \pm 0.11 , SJ-ACC3: control 1.02 \pm 0.12 , LEDP-M $1.74 \pm 0.43$; Fig. 5A). In contrast, miR-210 ratio was significantly altered in SW-13 after LEDP-M treatment $(0.32 \pm 0.06)$ compared with that in controls $(1.20 \pm 0.31)$, indicating elevated circulating miR-210 levels. Such an effect was not evident for SJ-ACC3 (control 1.26 \pm 0.74 , LEDP-M 0.83 \pm 0.32 ; Fig. 5B).

\section{Clinical data}

Six patients had been treated on a compassionate-use basis with a liposomally modified EDP-M scheme (lipEDP-M) including at least one liposomal drug. Although none of these heavily pretreated patients experienced an objective tumor response, the drug regimens were well tolerated. In three patients, kidney function was clearly impaired partly due to the renal toxicity of EDP-M, but the glomerular filtration rate did not further deteriorate for liposomal drug regimens. However, one patient experienced acute kidney failure after the second cycle
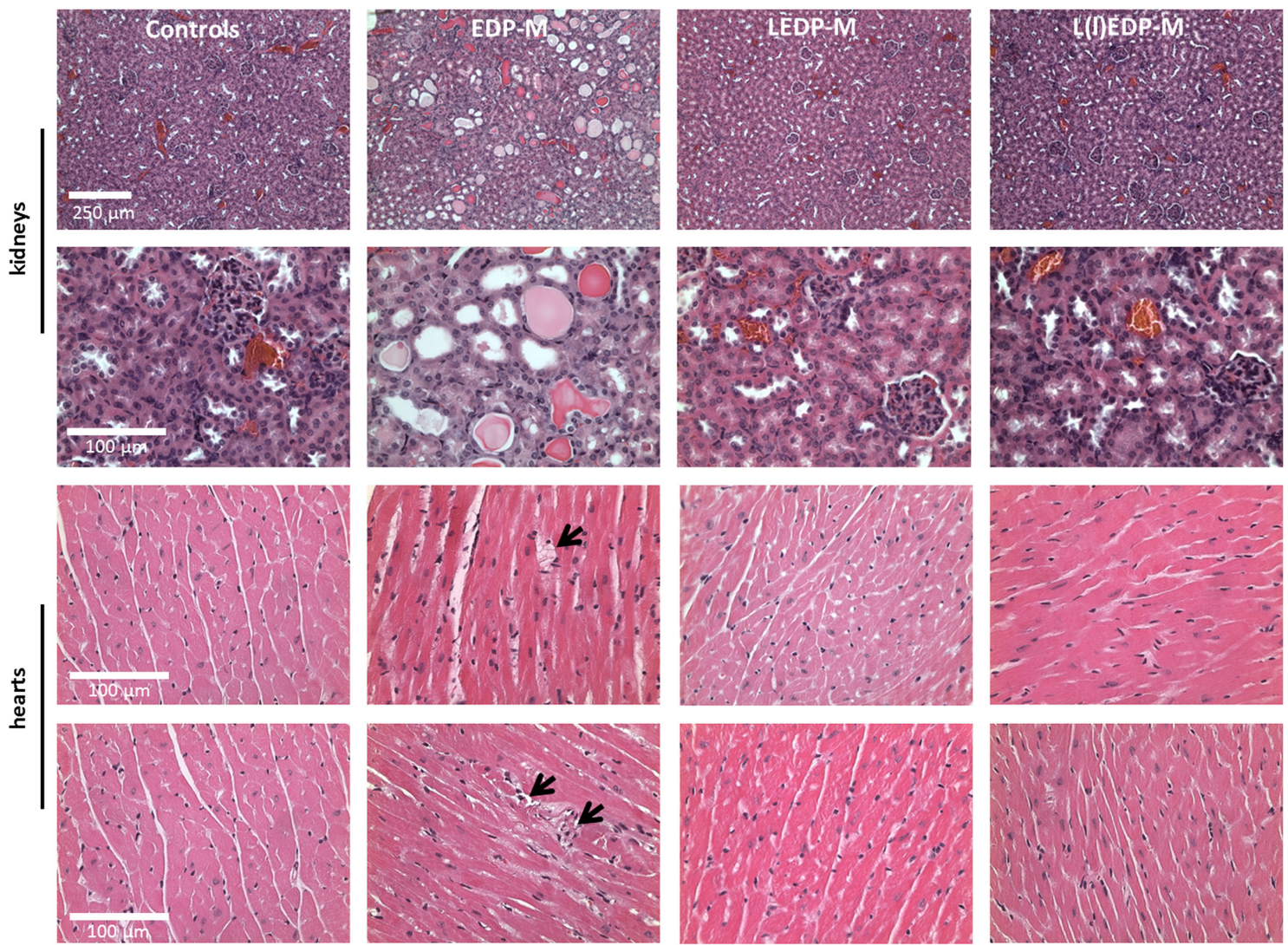

Figure 4

Pathological examination of HE-stained kidneys and hearts from the different treatment groups: control (NaCl), EDP-M, LEDP-M and L(I)EDP-M. Black arrows indicate vacuole formation and single-cell necrosis in hearts affected by EDP-M treatment. 
A

SW-13

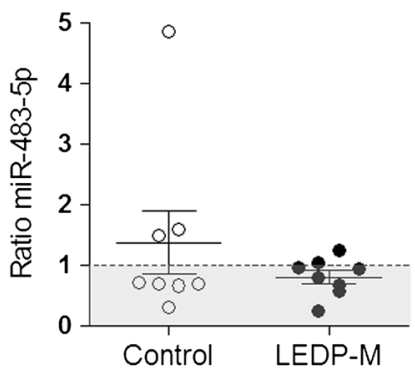

B

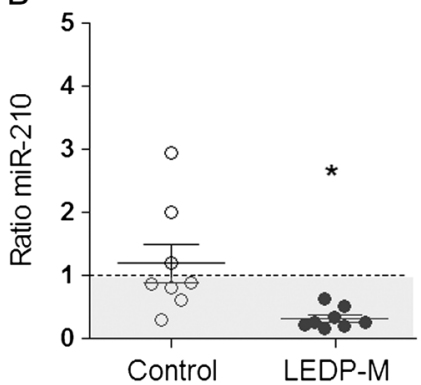

SJ-ACC3
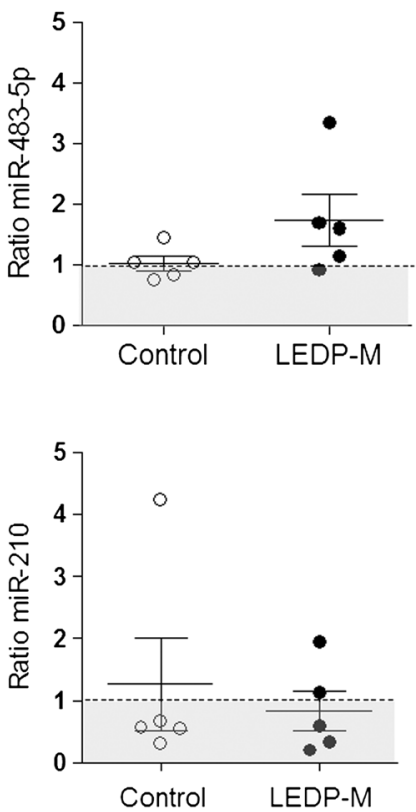

7

\section{Figure 5}

Quantitative real-time PCR analysis of intratumoral and circulating miR 483-5p (A) and miR-210 (B) of control (NaCl) and LEDP-M-treated SW-13 and SJ-ACC3 tumor-bearing mice. For analysis of changes upon therapeutic treatment in individual animals, the ratio of intratumoral to circulating miR level was calculated after normalization to controls. A value of 1 indicates unchanged equal levels in tumor and plasma. A value of $>1$ reveals an elevated expression of intratumoral $\mathrm{miR}$, whereas a ratio of $<1$ indicates elevated expression of circulating miR. Stars denote significant differences $(* P<0.05)$. of lipEDP-M, which was most likely due to sepsis. Of note, three of the six patients judged the modified regimen better tolerable than the previously administered conventional EDP-M scheme. Two patients mentioned no difference and one had no subjective toxicity at all upon EDP-M and lipEDP-M treatment (Table 1).

\section{Discussion}

EDP-M is currently the medical standard treatment for advanced ACC not amenable for surgery. Nevertheless, therapeutic efficacy in clinics is considerably complicated by patient heterogeneity and especially by dose-limiting adverse effects of this multi-chemotherapeutic treatment (Berruti et al. 2005, Fassnacht et al. 2012, 2013). Recently, we brought preclinical evidence for promising antitumoral potential and improved off-target profiles for LEDP-M in the classical, preclinical tumor model for ACC NCI-H295R (Hantel et al. 2014). To obtain the preclinical results with the highest clinically predictive power, we investigated liposomal schemes of EDP-M in two further tumor models for ACC in vivo: hormonally inactive SW-13 (Leibovitz et al. 1973) and pediatric SJ-ACC3 xenografts (Pinto et al. 2013). In this context, it has to be mentioned that origin and applicability of SW-13 as ACC tumor model is controversial as SW-13 was originally reported as derived from a small-cell carcinoma metastasized to the adrenal gland. Thereby, its adrenocortical origin has been questioned. However, due to the lack of ACC tumor intratumoral miR higher

equal miR level

circulating miR higher models representing the sub-entity of non-secreting ACC in preclinical experiments, SW-13 cells have been used in the past and in this study to complement preclinical experiments.

Even though all treatment arms demonstrated therapeutic efficacy against SW-13 xenografts, this study revealed the highest anti-tumoral effects for $\mathrm{L}(\mathrm{l})$ EDP-M. Overall, for L(l)EDP-M we detected a highly significant reduction in the total number of tumor cells and induction of necrosis upon one therapeutic cycle as well as significantly reduced tumor sizes upon repeated treatment cycles. Thereby, our previous study on NCI-H295R xenografts (Hantel et al. 2014) and this study predict for adult ACCs at least comparable or improved therapeutic efficacies of liposomal EDP-M schemes compared with the classical regime. Furthermore, acute and long-term complications of EDP-M are of main impact for clinical tolerability and therapeutic benefit. EDP-M leads to typical off-target profiles characterized by hematological toxicities with dose-limiting leukopenia, gastrointestinal and other toxicities including cardiotoxic and nephrotoxic events (Berruti et al. 2005, Fassnacht et al. 2012). After cytostatic treatment with doxorubicin, approximately one of four patients experiences congestive heart failure when cumulative doses exceed $500 \mathrm{mg} / \mathrm{m}^{2}$ (Lipshultz et al. 1991, Rahman et al. 2007). More recent data suggest the development of cardiomyopathy after anthracycline-based chemotherapy not only after much lower cumulative doses but also after 4-20 years in almost

Published by Bioscientifica Ltd 
25\% of patients (Steinherz et al. 1991, Hequet et al. 2004). This is of decisive importance for adult patients as well as for children as cytotoxic effects of anthracyclines are thought to be irreversible (Rahman et al. 2007). Accordingly, classical EDP-M treatment leads in these organs to clearly detectable pathological alterations, which are known to be common adverse effects of doxorubicin and cisplatin treatment as they have been intensively studied (Mettler et al. 1977, Devarajan et al. 2004, Rahman et al. 2007, Stathopoulos \& Boulikas 2012).

Consequently, we analyzed kidneys and hearts after multiple treatment cycles, as these organs are known to be the main off-target organs of cisplatin and doxorubicin treatment, respectively (Chlebowski 1979, Meyer \& Madias 1994). In accordance with the clinical situation, we detected severe pathological alterations in these organs after multiple therapeutic cycles with EDP-M. In recent years, liposomal doxorubicin and cisplatin have been successfully applied in clinics for a wide range of malignancies to overcome the severe adverse effects of their parenteral drugs and to further enhance treatment efficacy. Several clinical studies demonstrated that liposomal doxorubicin and cisplatin significantly reduced cardiotoxicity and nephrotoxicity in a variety of malignancies (Boulikas 2009, Marchal et al. 2015). Thus, a liposomal EDP-M scheme was very likely to improve off-target profiles and could therefore lead in clinical use to a better tolerated and highly efficient treatment option for advanced ACC. In accordance with these assumptions, we detected no comparable cardiotoxic and nephrotoxic impairment upon LEDP-M or L(l)EDP-M treatments even after up to four treatment cycles. Moreover, evaluation of achievement of study termination including an observation of adverse effects after multiple treatment cycles confirmed these results by demonstrating significantly prolonged survival after L(1)EDP-M treatment compared with the classical EDP-M protocol.

In contrast to NCI-H295R (Hantel et al. 2014) and SW-13, pediatric SJ-ACC3 xenografts showed slight therapeutic responsiveness upon one therapeutic cycle exclusively with the classical EDP-M treatment. However, none of the investigated treatment schemes led to an effective and sustained therapeutic response in a subsequent long-term study. The contribution of the multi-chemotherapeutic EDP-M regimen to the overall disease response is anyway controversial, and a recent preclinical study of Pinto and coworkers (2013) revealed that cisplatin, but not etoposide and doxorubicin as single agents, had potent anti-tumoral effects. Anti-tumor effects upon cisplatin were obtained by monotherapy using different dosing regimens and higher drug concentration. In addition, topotecan was identified as a potentially effective agent for pediatric ACC in this study. As outlined previously, liposomal cisplatin is already investigated in clinical studies (phase I, II and III trials); liposomal topotecan is also under preclinical evaluation for other malignancies (Tardi et al. 2000, Yu et al. 2012). Thus, a combinatorial approach of liposomal formulations of cisplatin and topotecan might be an interesting strategy to improve the therapeutic benefit and tolerability for pediatric ACC in the future.

However, not only prolonged survival time and general tolerability but also quality of life remains of pivotal interest for patients. ACC is a heterogeneous disease, which is often highly aggressive and progressive, but sometimes also appears more indolent and slowly proceeding. Responses upon EDP-M treatment can range from no anti-tumoral effect to high treatment efficacy (Berruti et al. 2005, Fassnacht et al. 2012). As biomarkers for treatment response do not exist yet, such high variability in therapeutic outcome makes it almost impossible to weigh up patient's long-term benefit and life quality against multiple chemotherapeutic cycles with putative severe side effects (Ferrari et al. 2016). Analysis of microRNA (miR) draws increasing attention as miR represents important regulators for pathological settings and tumorigenesis (Cherradi 2015). Aberrant miR expression patterns have been demonstrated for a variety of cancers including ACC, and circulating miRs have been correlated with cancer progression, therapy response and outcome of survival in blood samples (Brase et al. 2010, Cherradi 2015). Assessing the potential of miR-483-5p and miR-210 as therapeutic biomarkers, we evaluated the ratio of intratumoral to circulating $\mathrm{miR}$ to detect individual treatment-dependent changes. The MIR483 gene is located in the second intron of the IGF2 gene, and high miR-483-5p could be correlated with high expression of IGF2 and malignancy in ACC (Patterson et al. 2011). Hypoxia-inducible miR-210 is involved in proliferation, mitochondrial respiration, DNA repair and angiogenesis (Huang et al. 2010), and high miR-210 levels were correlated with clinicopathologic parameters of aggressiveness as well as poor prognosis in ACC (Duregon et al. 2014) and other tumor entities (Greither et al. 2010, 2012, Hong et al. 2012, Qiu et al. 2013).

SW-13 and SJ-ACC3 tumors and plasma exosomes were analyzed after one therapeutic cycle upon $\mathrm{NaCl}$ or LEDP-M treatment. Our miR analysis was basically built on the hypothesis that tumor response to a given therapy might lead to specific changes in either intratumoral

Published by Bioscientifica Ltd. 
or circulating levels of specific microRNAs. A few of publications describing the analysis of microRNA in patient tumors and plasma samples are available for ACC (Cherradi 2015), but to our knowledge a possible correlation of therapeutic efficacy has never been tested before. Existing miR data in ACC patients often describe synchronous upregulation or downregulation of miRs in tumor and plasma samples (Cherradi 2015). However, these results are based on the comparison of healthy individuals with tumor-bearing individuals. Regarding other tumor entities including breast, NSCLC, colorectal, prostate and gastric cancer, an assessment of therapeutic treatment efficacy and miR levels in serum or plasma samples has been initiated, and these publications conclude that a correlation of therapeutic efficacy with the circulating miR level might exist (Kim et al. 2011, Jung et al. 2012, Li et al. 2013, Kjersem et al. 2014, Lin et al. 2014, Muller et al. 2014). To take all putative regulations into account, we defined the miR ratio. A ratio of 1 indicates unchanged levels in tumor and plasma, a value of $>1$ reveals elevated levels of intratumoral and a ratio of $<1$ reveals an increase in circulating miR. In our experiments, we detected a significant decrease of miR ratio for miR-210 indicating a significant increase in circulating miR levels specifically in the LEDP-M-responsive SW-13 tumor model, whereas no comparable effects were detectable for therapy-resistant SJ-ACC3. Thus, our initial data provide evidence that an increase in circulating miR-210 might be an interesting candidate as marker for tumor response. However, to our knowledge, general interactions of circulating miRs in plasma and commercial liposomal formulations have not been evaluated yet. Additional investigations are needed to assess the general potential of miR-210 as a therapeutic biomarker for ACC.

Despite such promising research approaches for the future, liposomal EDP-M has great potential to instantly improve the therapeutic situation in clinics. Our first experience with liposomal cytotoxic drugs in ACC patients demonstrated that these drugs are overall very well tolerated despite the fact that all patients were heavily pretreated with standard EDP and other cytotoxic drugs. The fact that no patient experienced objective tumor response has to be seen in the context of very advanced disease and EDP-M pre-treatment, which both might make drug resistance more likely.

In summary, we conclude that liposomally modified EDP-M holds potential to instantly improve the current medical treatment of ACC. Although liposomal etoposide as applied in L(l)EDP-M has not been introduced in clinical practice yet, our liposomal treatment protocols contain liposomal cisplatin (Lipoplatin) and doxorubicin (Caelyx and Myocet), which are for other tumor entities already in clinical use (Boulikas 2009, Marchal et al. 2015). Consequently, their implementation would allow a fast clinical translation. Thereby, our findings could improve the therapeutic efficacy, but mainly also tolerability and quality of life for advanced ACC patients.

\section{Declaration of interest}

The authors declare that there is no conflict of interest that could be perceived as prejudicing the impartiality of the research reported.

\section{Funding}

This work was supported by Wilhelm-Sander-Stiftung to Constanze Hantel and Felix Beuschlein (2011.003.2), ESE short-term fellowship grant and Seventh Framework Program (FP7/2007-2013) under grant agreement no. 259735. Lipoplatin and mitotane were kindly provided by Teni Boulikas (Regulon Inc.) and HRA Pharma, respectively. MicroRNA studies were funded by the Hungarian National Research, Development and Innovation Office - NKFIH (K115398 to Peter Igaz).

\section{Acknowledgements}

The authors are indebted to Igor Shapiro and Susanne Mentz for their excellent technical support. Furthermore, the authors thank Chris Morton (St Jude Children's Research Hospital, Memphis, TN, USA).

\section{References}

Assie G, Letouze E, Fassnacht M, Jouinot A, Luscap W, Barreau O, Omeiri H, Rodriguez S, Perlemoine K, Rene-Corail F, et al. 2014 Integrated genomic characterization of adrenocortical carcinoma. Nature Genetics 46 607-612. (doi:10.1038/ng.2953)

Berruti A, Terzolo M, Sperone P, Pia A, Della Casa S, Gross DJ, Carnaghi C, Casali P, Porpiglia F, Mantero F, et al. 2005 Etoposide, doxorubicin and cisplatin plus mitotane in the treatment of advanced adrenocortical carcinoma: a large prospective phase II trial. Endocrine-Related Cancer 12 657-666. (doi:10.1677/ erc.1.01025)

Berruti A, Baudin E, Gelderblom H, Haak HR, Porpiglia F, Fassnacht M, Pentheroudakis G \& Group EGW 2012 Adrenal cancer: ESMO clinical practice guidelines for diagnosis, treatment and follow-up. Annals of Oncology 23 (Supplement 7) vii131-138. (doi:10.1093/ annonc/mds231)

Boulikas T 2009 Clinical overview on Lipoplatin: a successful liposomal formulation of cisplatin. Expert Opinion on Investigational Drugs $\mathbf{1 8}$ 1197-1218. (doi:10.1517/13543780903114168)

Brase JC, Wuttig D, Kuner R \& Sultmann H 2010 Serum microRNAs as non-invasive biomarkers for cancer. Molecular Cancer 9306. (doi:10.1186/1476-4598-9-306)

Cherradi N 2015 microRNAs as potential biomarkers in adrenocortical cancer: progress and challenges. Frontiers in Endocrinology 6195. (doi:10.3389/fendo.2015.00195)

Chlebowski RT 1979 Adriamycin (doxorubicin) cardiotoxicity: a review. Western Journal of Medicine 131 364-368.

Devarajan P, Tarabishi R, Mishra J, Ma Q, Kourvetaris A, Vougiouka M \& Boulikas T 2004 Low renal toxicity of lipoplatin compared to cisplatin in animals. Anticancer Research 24 2193-2200.

Duregon E, Rapa I, Votta A, Giorcelli J, Daffara F, Terzolo M, Scagliotti GV, Volante M \& Papotti M 2014 MicroRNA expression patterns in 
adrenocortical carcinoma variants and clinical pathologic correlations. Human Pathology 45 1555-1562. (doi:10.1016/j.humpath. 2014.04.005)

Fassnacht M, Terzolo M, Allolio B, Baudin E, Haak H, Berruti A, Welin S, Schade-Brittinger C, Lacroix A, Jarzab B, et al. 2012 Combination chemotherapy in advanced adrenocortical carcinoma. New England Journal of Medicine 366 2189-2197. (doi:10.1056/NEJMoa1200966)

Fassnacht M, Kroiss M \& Allolio B 2013 Update in adrenocortical carcinoma. Journal of Clinical Endocrinology and Metabolism 98 4551-4564. (doi:10.1210/jc.2013-3020)

Fassnacht M, Berruti A, Baudin E, Demeure MJ, Gilbert J, Haak H, Kroiss M, Quinn DI, Hesseltine E, Ronchi CL, et al. 2015 Linsitinib (OSI-906) versus placebo for patients with locally advanced or metastatic adrenocortical carcinoma: a double-blind, randomised, phase 3 study. Lancet Oncology 16 426-435. (doi:10.1016/S14702045(15)70081-1)

Ferrari L, Claps M, Grisanti S \& Berruti A 2016 Systemic therapy in locally advanced or metastatic adrenal cancers: a critical appraisal and clinical trial update. European Urology Focus 1 298-300. (doi:10.1016/j.euf.2015.06.005)

Gabizon A, Shmeeda H \& Grenader T 2012 Pharmacological basis of pegylated liposomal doxorubicin: impact on cancer therapy. European Journal of Pharmaceutical Sciences 45 388-398. (doi:10.1016/j.ejps.2011.09.006)

Greither T, Grochola LF, Udelnow A, Lautenschlager C, Wurl P \& Taubert H 2010 Elevated expression of microRNAs 155, 203, 210 and 222 in pancreatic tumors is associated with poorer survival. International Journal of Cancer 126 73-80. (doi:10.1002/ijc.24687)

Greither T, Wurl P, Grochola L, Bond G, Bache M, Kappler M, Lautenschlager C, Holzhausen HJ, Wach S, Eckert AW, et al. 2012 Expression of microRNA 210 associates with poor survival and age of tumor onset of soft-tissue sarcoma patients. International Journal of Cancer 130 1230-1235. (doi:10.1002/ijc.26109)

Hantel C \& Beuschlein F 2016 Xenograft models for adrenocortical carcinoma. Molecular and Cellular Endocrinology 421 28-33. (doi:10.1016/j.mce.2015.05.031)

Hantel C, Lewrick F, Schneider S, Zwermann O, Perren A, Reincke M, Suss R \& Beuschlein F 2010 Anti insulin-like growth factor I receptor immunoliposomes: a single formulation combining two anticancer treatments with enhanced therapeutic efficiency. Journal of Clinical Endocrinology and Metabolism 95 943-952. (doi:10.1210/jc.2009-1980)

Hantel C, Lewrick F, Reincke M, Suss R \& Beuschlein F 2012 Liposomal doxorubicin-based treatment in a preclinical model of adrenocortical carcinoma. Journal of Endocrinology 213 155-161. (doi:10.1530/JOE11-0427)

Hantel C, Jung S, Mussack T, Reincke M \& Beuschlein F 2014 Liposomal polychemotherapy improves adrenocortical carcinoma treatment in a preclinical rodent model. Endocrine-Related Cancer 21 383-394. (doi:10.1530/ERC-13-0439)

Hequet O, Le QH, Moullet I, Pauli E, Salles G, Espinouse D, Dumontet C, Thieblemont C, Arnaud P, Antal D, et al. 2004 Subclinical late cardiomyopathy after doxorubicin therapy for lymphoma in adults. Journal of Clinical Oncology 22 1864-1871. (doi:10.1200/JCO.2004.06.033)

Hong L, Yang J, Han Y, Lu Q, Cao J \& Syed L 2012 High expression of miR-210 predicts poor survival in patients with breast cancer: a meta-analysis. Gene 507 135-138. (doi:10.1016/j.gene.2012.07.025)

Huang X, Le QT \& Giaccia AJ 2010 MiR-210 - micromanager of the hypoxia pathway. Trends in Molecular Medicine 16 230-237. (doi:10.1016/j.molmed.2010.03.004)

Jung EJ, Santarpia L, Kim J, Esteva FJ, Moretti E, Buzdar AU, Di Leo A, Le XF, Bast RC Jr, Park ST, et al. 2012 Plasma microRNA 210 levels correlate with sensitivity to trastuzumab and tumor presence in breast cancer patients. Cancer 118 2603-2614. (doi:10.1002/ cncr.26565)
Kim CH, Kim HK, Rettig RL, Kim J, Lee ET, Aprelikova O, Choi IJ, Munroe DJ \& Green JE 2011 miRNA signature associated with outcome of gastric cancer patients following chemotherapy. BMC Medical Genomics 4 79. (doi:10.1186/1755-8794-4-79)

Kjersem JB, Ikdahl T, Lingjaerde OC, Guren T, Tveit KM \& Kure EH 2014 Plasma microRNAs predicting clinical outcome in metastatic colorectal cancer patients receiving first-line oxaliplatin-based treatment. Molecular Oncology 8 59-67. (doi:10.1016/j. molonc.2013.09.001)

Kroiss M, Quinkler M, Johanssen S, van Erp NP, Lankheet N, Pollinger A, Laubner K, Strasburger CJ, Hahner S, Muller HH, et al. 2012 Sunitinib in refractory adrenocortical carcinoma: a phase II, single-arm, open-label trial. Journal of Clinical Endocrinology and Metabolism 97 3495-3503. (doi:10.1210/jc.2012-1419)

Leibovitz A, McCombs WM 3rd, Johnston D, McCoy CE \& Stinson JC 1973 New human cancer cell culture lines. I. SW-13, small-cell carcinoma of the adrenal cortex. Journal of the National Cancer Institute 51 691-697.

Li ZH, Zhang H, Yang ZG, Wen GQ, Cui YB \& Shao GG 2013 Prognostic significance of serum microRNA-210 levels in nonsmall-cell lung cancer. Journal of International Medical Research 41 1437-1444. (doi:10.1177/0300060513497560)

Lin HM, Castillo L, Mahon KL, Chiam K, Lee BY, Nguyen Q, Boyer MJ, Stockler MR, Pavlakis N, Marx G, et al. 2014 Circulating microRNAs are associated with docetaxel chemotherapy outcome in castrationresistant prostate cancer. British Journal of Cancer 110 2462-2471. (doi:10.1038/bjc.2014.181)

Lipshultz SE, Colan SD, Gelber RD, Perez-Atayde AR, Sallan SE \& Sanders SP 1991 Late cardiac effects of doxorubicin therapy for acute lymphoblastic leukemia in childhood. New England Journal of Medicine 324 808-815. (doi:10.1056/NEJM199103213241205)

Malandrino P, Al Ghuzlan A, Castaing M, Young J, Caillou B, Travagli JP, Elias D, de Baere T, Dromain C, Paci A, et al. 2010 Prognostic markers of survival after combined mitotane- and platinum-based chemotherapy in metastatic adrenocortical carcinoma. Endocrine-Related Cancer 17 797-807. (doi:10.1677/ERC-09-0341)

Marchal S, El Hor A, Millard M, Gillon V \& Bezdetnaya L 2015 Anticancer drug delivery: an update on clinically applied nanotherapeutics. Drugs 75 1601-1611. (doi:10.1007/s40265-015-0453-3)

Mettler FP, Young DM \& Ward JM 1977 Adriamycin-induced cardiotoxicity (cardiomyopathy and congestive heart failure) in rats. Cancer Research 37 2705-2713.

Meyer KB \& Madias NE 1994 Cisplatin nephrotoxicity. Mineral and Electrolyte Metabolism 20 201-213.

Muller V, Gade S, Steinbach B, Loibl S, von Minckwitz G, Untch M, Schwedler K, Lubbe K, Schem C, Fasching PA, et al. 2014 Changes in serum levels of miR-21, miR-210, and miR-373 in HER2-positive breast cancer patients undergoing neoadjuvant therapy: a translational research project within the Geparquinto trial. Breast Cancer Research and Treatment 147 61-68. (doi:10.1007/s10549-014-3079-3)

Nagy Z, Baghy K, Hunyadi-Gulyas E, Micsik T, Nyiro G, Racz G, Butz H, Perge P, Kovalszky I, Medzihradszky KF, et al. 2015 Evaluation of 9-cis retinoic acid and mitotane as antitumoral agents in an adrenocortical xenograft model. American Journal of Cancer Research $53645-3658$

Patterson EE, Holloway AK, Weng J, Fojo T \& Kebebew E 2011 MicroRNA profiling of adrenocortical tumors reveals miR-483 as a marker of malignancy. Cancer 117 1630-1639. (doi:10.1002/ cncr.25724)

Pinto EM, Morton C, Rodriguez-Galindo C, McGregor L, Davidoff AM, Mercer K, Debelenko LV, Billups C, Ribeiro RC \& Zambetti GP 2013 Establishment and characterization of the first pediatric adrenocortical carcinoma xenograft model identifies topotecan as a potential chemotherapeutic agent. Clinical Cancer Research 19 1740-1747. (doi:10.1158/1078-0432.CCR-12-3354) http://erc.endocrinology-journals.org

DOI: 10.1530/ERC-16-0249
C 2016 Society for Endocrinology Printed in Great Britain
Published by Bioscientifica Ltd 
Qiu S, Lin S, Hu D, Feng Y, Tan Y \& Peng Y 2013 Interactions of miR323/miR-326/miR-329 and miR-130a/miR-155/miR-210 as prognostic indicators for clinical outcome of glioblastoma patients. Journal of Translational Medicine 11 10. (doi:10.1186/1479-5876-11-10)

Rahman AM, Yusuf SW \& Ewer MS 2007 Anthracycline-induced cardiotoxicity and the cardiac-sparing effect of liposomal formulation. International Journal of Nanomedicine 2 567-583.

Ronchi CL, Sbiera S, Kraus L, Wortmann S, Johanssen S, Adam P, Willenberg HS, Hahner S, Allolio B \& Fassnacht M 2009 Expression of excision repair cross complementing group 1 and prognosis in adrenocortical carcinoma patients treated with platinum-based chemotherapy. Endocrine-Related Cancer 16 907-918. (doi:10.1677/ ERC-08-0224)

Schmittgen TD \& Livak KJ 2008 Analyzing real-time PCR data by the comparative C(T) method. Nature Protocols 3 1101-1108. (doi:10.1038/nprot.2008.73)

Stathopoulos GP \& Boulikas T 2012 Lipoplatin formulation review article. Journal of Drug Delivery 2012581363. (doi:10.1155/2012/581363)
Steinherz LJ, Steinherz PG, Tan CT, Heller G \& Murphy ML 1991 Cardiac toxicity 4 to 20 years after completing anthracycline therapy. JAMA 266 1672-1677. (doi:10.1001/jama.1991. $03470120074036)$

Tardi P, Choice E, Masin D, Redelmeier T, Bally M \& Madden TD 2000 Liposomal encapsulation of topotecan enhances anticancer efficacy in murine and human xenograft models. Cancer Research 60 3389-3393.

Yu Y, Wang ZH, Zhang L, Yao HJ, Zhang Y, Li RJ, Ju RJ, Wang XX, Zhou J, Li N, et al. 2012 Mitochondrial targeting topotecan-loaded liposomes for treating drug-resistant breast cancer and inhibiting invasive metastases of melanoma. Biomaterials 33 1808-1820. (doi:10.1016/j.biomaterials. 2011.10.085)

Zheng S, Cherniack AD, Dewal N, Moffitt RA, Danilova L, Murray BA, Lerario AM, Else T, Knijnenburg TA, Ciriello G, et al. 2016 Comprehensive Pan-Genomic characterization of adrenocortical carcinoma. Cancer Cell 29 723-736. (doi:10.1016/j.ccell. 2016.04.002)

Received in final form 18 August 2016

Accepted 22 August 2016

Accepted Preprint published online 22 August 2016
Published by Bioscientifica Ltd. 